\title{
New Heuristic-Based Design of Robust Power System Stabilizers
}

\author{
S. Dechanupaprittha, I. Ngamroo, K. Pasupa, J. Tippayachai, \\ K. Hongesombut, Member, IEEE, and Y. Mitani, Member, IEEE
}

\begin{abstract}
This paper proposes a new robust design of power system stabilizers (PSSs) in a multimachine power system using a heuristic optimization method. The structure of each PSS used is similar to that of a conventional lead/lag stabilizer. The proposed design regards a multimachine power system with PSSs as a multi-input multi-output (MIMO) control system. Additionally, a multiplicative uncertainty model is taken into account in the power system representation. Accordingly, the robust stability margin can be guaranteed by a multiplicative stability margin (MSM). The presented method utilizes the MSM as the design specification for robust stability. To acquire the control parameters of PSSs, a control design in MIMO system is formulated as an optimization problem. In the selection of objective function, not only disturbance attenuation performance but also robust stability indices are considered. Subsequently, the hybrid tabu search and evolutionary programming (Hybrid TS/EP) is employed to search for the optimal parameters. The significant effects of designed PSSs are investigated under several system operating conditions.
\end{abstract}

Index Terms - Robust control, tabu search, evolutionary programming, optimization problems, multivariable control system, power system stabilizer.

\section{INTRODUCTION}

The insufficient damping of an electromechanical oscillation mode (EMO) always causes an unavoidable low frequency oscillation $(0.2-2.5 \mathrm{~Hz})$ in a power system. To solve this problem, a power system stabilizer (PSS) is regarded as a significantly effective device to increase the damping of EMO mode via an excitation system [1]. In addition, the structure of a conventional PSS (CPSS) is a lead/lag controller with speed or electrical power deviations input. Therefore, it is easy to implement in practice.

However, the main problem of CPSS is the parameter tuning of lead/lag controllers. Many heuristic approaches have been successfully applied to achieve the control parameters, such as tabu search [2], genetic algorithm [3] and simulated annealing [4]. In these studies, however, the uncertainty model was not embedded in the mathematical model of the power system. Furthermore, the robust stability against system uncertainties of PSS was not considered in the optimization process. Therefore, the robust stability margin of PSSs mentioned above may not be guaranteed in the face of uncertainties.

S. Dechanupaprittha, I. Ngamroo, K. Pasupa, and, J. Tippayachai, are with the Electrical Power Engineering Program, Sirindhorn International Institute of Technology (SIIT), Thammasat University, Pathumthani 12121, Thailand (e-mail: sanchai@ thaimobilis.com; ngamroo@siit.tu.ac.th kitsuchart@hotmail.com; jarurote@siit.tu.ac.th).

K. Hongesombut and Y. Mitani are with the Electrical Engineering Program, Kyushu Institute of Technology, Kitakyushu, Fukuoka 804-8550, Japan (e-mail: komsan@ele.kyutech.ac.jp; mitani@ele.kyutech.ac.jp).
In this paper, a new robust design of PSSs in a multi machine power system is proposed. The presented method translates the design problem to a multi-input multi-output (MIMO) control system. In addition, effects of system nonlinearity such as modelling and characteristics of synchronous machines, system loading conditions, variations in power system configuration due to unpredictable disturbances, etc. are treated as system uncertainties. Accordingly, system uncertainties represented by a multiplicative uncertainty model are incorporated in the mathematical model of the study system. As a result, a robust design of MIMO system can be formulated as an optimization problem. The control parameters of PSSs are optimized by the hybrid tabu search and evolutionary programming (Hybrid TS/EP). In the objective function, not only the disturbance attenuation performance but also robust stability indices are included. Based on the applied uncertainty model, the multiplicative stability margin (MSM) can be regarded as the design specification. The evaluation of control effects are carried out in the case of two-area fourmachine power system.

The organization of this paper is as follows. Section II explains the proposed design method. Next, the Hybrid TS/EP algorithm is given in section III. Subsequently, the evaluation study is shown in section IV. Finally, the outcomes from this paper are summarized.

\section{Control Problem Formulation}

\section{A. Linearized Model of Power System}

The study power system consists of 4 generators in 2 areas as illustrated in Fig. 1, [5]. It is represented by a linearized model around a designed operation point. Each generator is equipped with a simplified exciter and is represented by a 5state transient model.

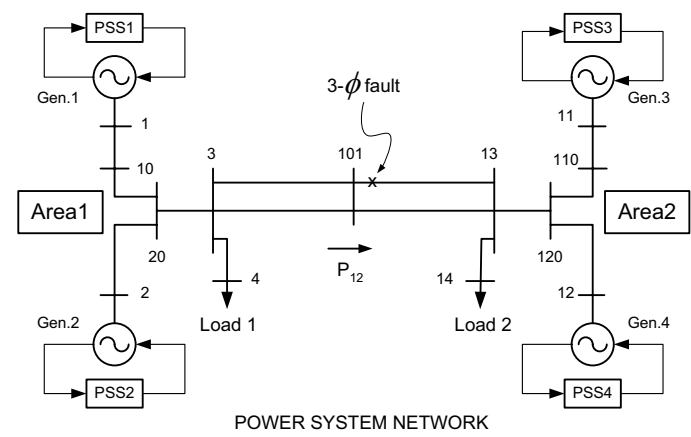

Fig. 1. A single-line diagram for the two-area four-machine power system. 
The state equations of a linearized power system $\left(A_{S}, B_{S}\right.$, $\left.C_{S}, D_{S}\right)$ in Fig. 1 can be expressed as:

$$
\begin{aligned}
\Delta \dot{x} & =A_{S} \Delta x+B_{S} \Delta u_{P S S} \\
\Delta y & =C_{S} \Delta x+D_{S} \Delta u_{P S S} \\
\Delta u_{P S S} & =K_{P S S}(s) \Delta \omega
\end{aligned}
$$

where, $\Delta x=\left[\begin{array}{lllll}\Delta \delta & \Delta \omega & \Delta e_{d}^{\prime} & \Delta e_{q}^{\prime} & \Delta E_{f d}\end{array}\right]^{T},(5 n \times 1)$; $\Delta y=\Delta \omega,(m \times 1) ; \Delta \delta$ denotes the deviation of rotor angle, $(n \times 1) ; \Delta \omega$ is the deviation of rotor speed, $(n \times 1) ; \Delta e_{d}^{\prime}$ and $\Delta e_{q}^{\prime}$ are the deviations of transient internal voltages of a generator in $d$-axis and $q$-axis, respectively, $(n \times 1) ; \Delta E_{f d}$ is the deviation of field voltage, $(n \times 1) ; K_{P S S}(s)$ is the diagonal controller with designed PSSs as diagonal elements, $(m \times m)$; $\Delta u_{P S S}$ is the control output signal of $K_{P S S}(s),(m \times 1) ; n$ and $m$ are the numbers of machines and PSSs, respectively. Note that the system (1) is an MIMO control system and is referred to as the nominal plant $G$.

\section{B. PSS Structure}

The transfer function of PSS is in a form of a lead/lag controller as:

$$
\begin{aligned}
\Delta u_{P S S, i}= & K_{i} \cdot \frac{s T_{w}}{1+s T_{w}} \cdot \frac{1+s T_{1 i}}{1+s T_{2 i}} \cdot \frac{1+s T_{3 i}}{1+s T_{4 i}} \cdot \Delta \omega_{i}, \\
& i=1, \ldots, m,
\end{aligned}
$$

where, $\Delta u_{P S S, i}$ and $\Delta \omega_{i}$ are the control output signal and the rotor speed deviation at the $i$-th machine, respectively; $K_{i}$ is a controller gain; $T_{w}$ is a wash-out time constant (s); and $T_{1 i}$, $T_{2 i}, T_{3 i}$, and $T_{4 i}$ are time constants (s).

In this paper, $T_{w}$ is set to $10 \mathrm{~s}$. The control parameters $K_{i}, T_{1 i}, T_{2 i}, T_{3 i}$, and $T_{4 i}$ are searched based on the objective function explained in the following subsection.

\section{Determination of Objective Function}

In derivation of the objective function, both attenuation performance of system disturbance and robust stability of control system against system uncertainties are taken into consideration. Since the main purpose of the PSS control is to improve the system damping following any disturbances, therefore, the damping ratio $(\zeta)$ of EMO mode is used as a design specification. Assuming that eigenvalues corresponding to the mode of oscillation can be determined as $-\sigma \pm j \omega_{d}$, the damping ratio is given by:

$$
\zeta_{\text {actual }}=\frac{-\sigma}{\sqrt{\sigma^{2}+\omega_{d}^{2}}} .
$$

The desired damping ratio of the eigenvalues corresponding to the mode of oscillation is specified as $\zeta_{\text {desired }}$. Accordingly, the difference between the desired and the actual damping ratios can be defined as:

$$
\psi=\left|\zeta_{\text {desired }}-\zeta_{\text {actual }}\right| .
$$

Note that the disturbance attenuation performance increases when $\psi$ is minimized.

Here, the D-Stability region is exploited to guarantee the desired damping ratio and the real part of controlled EMO

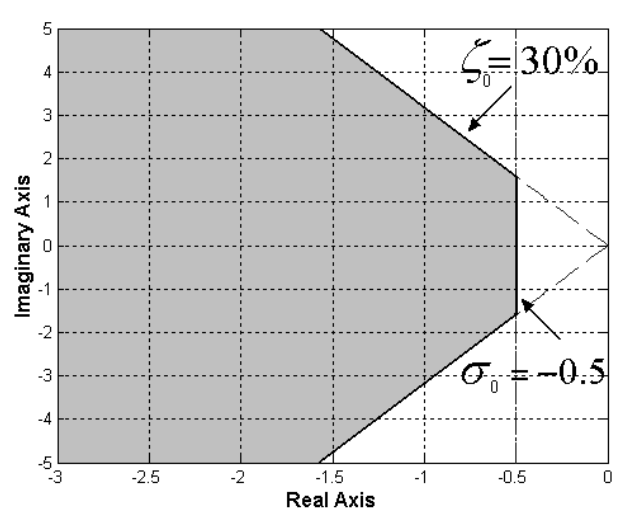

Fig. 2. D-Stability Region.

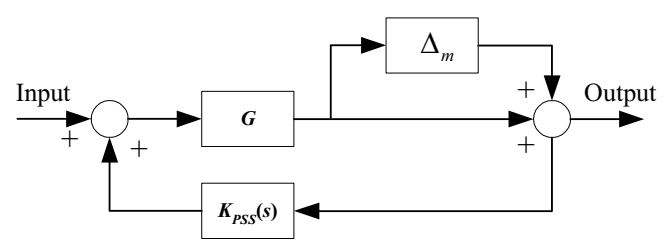

Fig. 3. Feedback system with multiplicative uncertainty.

mode. As shown in Fig. 2, $\zeta_{0}$ and $\sigma_{0}$ are set at $30 \%$ and -0.5 , respectively.

For the robust stability of a system, the plant uncertainty is modelled as a multiplicative form [6] demonstrated in Fig. 3. $\Delta_{m}$ is a stable multiplicative uncertainty. Based on the smallgain theorem, the closed loop system will be robustly stable if

$$
\left|\Delta_{m}\right|<\frac{1}{\left|G \cdot K_{P S S}\left(1-G \cdot K_{P S S}\right)^{-1}\right|},
$$

where, the symbol $|\bullet|$ shows the magnitude of transfer function $(\bullet)$. Note that $G \cdot K_{P S S}\left(1-G \cdot K_{P S S}\right)^{-1}$ is the complementary sensitivity function, $T$. Based on this uncertainty representation, the robust stability margin can be guaranteed in term of MSM. In other words, MSM also implies the maximum uncertainty bound and can be calculated by

$$
\mathrm{MSM}=\frac{1}{\|T\|_{\infty}},
$$

where, $\|T\|_{\infty}$ is the $\infty$-norm of $T$. From (5) and (6), it is clear that by minimizing $\|T\|_{\infty}$, the MSM increases and the robust stability will be ensured [6]. Thus, the normalized robustness index of the objective function is defined as:

$$
\gamma=\|T\|_{\infty} /\|T\|_{\infty(\text { initial })},
$$

where, $\|T\|_{\infty \text { (initial) }}$ is the $\infty$-norm of $T$ at the initial of a search process.

Combining (4) and (7), the control problem can be formulated as the following optimization problem:

$$
\begin{array}{cl}
\text { Minimize } & C\left(K_{i}, T_{j i}\right)=\psi+\gamma, \\
\text { subject to } & K_{\min } \leq K_{i} \leq K_{\max }, \quad i=1, \ldots, m \\
& T_{i, \min } \leq T_{j i} \leq T_{i, \max }, \quad j=1, \ldots, 4
\end{array}
$$


where, $C\left(K_{i}, T_{j i}\right)$ is the objective function. The minimum and maximum values of the gain $K_{i}$ are set as 1 and 500, respectively. The minimum and maximum values of the time constants $T_{j i}$ are set as 0.01 and 1 , respectively. In this paper, the hybrid tabu search and evolutionary programming method (Hybrid TS/EP) introduced by [7] is applied to solve the optimization problem.

\section{Hybrid TABU SEARCH AND EVOlUtionary PROGRAMMING METHOd (HYBRID TS/EP)}

\section{A. Initialization}

The actual parameters of the $N$ parameters, $P_{i}, i=1, \ldots$, $N$, are randomly generated between the minimum actual parameters $\left(P_{i, \min }\right)$ and the maximum actual parameters $\left(P_{i, \max }\right)$ of the $k$-th individual solution, $X_{k}=\left[P_{1}^{k}, \ldots, P_{i}^{k}, \ldots, P_{N}^{k}\right]$, $k=1, \ldots, N P$, where $N P$ is the population size. After initializing the individual solutions in the population, the objective function in (8) is used to verify the quality of initial individuals. The best initial individual is recorded into the tabu list (to be discussed in Subsection III-C).

Furthermore, for each generation, Hybrid TS/EP performs 5 operations: perturbation strategies, tabu list restriction, fitness function evaluation, rank selection with elitism, and adaptive parameter setting strategies (to be discussed in Subsection IIIB, III-C, III-D, and III-E, respectively).

\section{B. Perturbation Strategies}

In this paper, hybrid TS/EP use mutation as a diversification searching strategy and arithmetic crossover [8] operator as an intensification searching strategy to obtain the optimal or near optimal solution [7].

1) Mutation: The offspring individuals (the new trial solutions) obtained from mutation process, $X_{k}^{\text {new }}=$ $\left[P_{1}^{k, n e w}, \ldots, P_{i}^{k, n e w}, \ldots, P_{N}^{k, n e w}\right], k=1, \ldots, N_{m}$, are defined as:

$$
P_{i}^{k, \text { new }}=P_{i}^{k}+H\left(0, \mu_{i}\right), \quad i=1, \ldots, N,
$$

where,

\begin{tabular}{|c|c|}
\hline$P_{i}^{k}:$ & $\begin{array}{l}\text { the } i \text {-th actual parameter of } k \text {-th parent indi- } \\
\text { vidual; }\end{array}$ \\
\hline$H\left(0, \mu_{i}\right)$ : & a uniform random variable with variance, $\mu_{i}$; \\
\hline & $\left|C_{k} / C_{\max }\right|\left(P_{i, \max }-P_{i, \min }\right) \beta, i=1, \ldots, N$ \\
\hline$C_{k}:$ & $\begin{array}{l}\text { the objective value of the } k \text {-th parent individ- } \\
\text { ual; }\end{array}$ \\
\hline$C_{\max }:$ & $\begin{array}{l}\text { the maximum objective value in the parent } \\
\text { population; }\end{array}$ \\
\hline & an adaptive mutation scale; \\
\hline & the number of mutated individuals. \\
\hline
\end{tabular}

Note if $P_{i}^{k, n e w}$ is higher or lower than its operating limits, set it to the limit.

2) Arithmetic Crossover: Based on the inherited genotypes of two randomly selected parent individuals $X_{k 1}$ and $X_{k 2}$, the offspring individuals obtained from the arithmetic crossover, $X_{k}^{\text {new }}=\left[P_{1}^{k, \text { new }}, \ldots, P_{i}^{k, \text { new }}, \ldots, P_{N}^{k, \text { new }}\right], k=N_{m}+$ $1, \ldots, N P$, are formulated by:

$$
X_{k}^{n e w}=X_{k 1}+u \cdot\left(X_{k 2}-X_{k 1}\right),
$$

where, $u$ is defined as a uniform random number ranges from zero to one.

\section{Tabu List Restriction}

The tabu list (TL) is used to keep best offspring individuals (solution vectors) in past iterations. During the search process, a new solution vector enters TL and the oldest one is released, as shown in Fig. 4. In particular, this technique prevents a cycling of visited offspring individuals in the perturbation process by forbidding perturbed individuals, which are similar to those in TL, from being used as solution candidates [9].

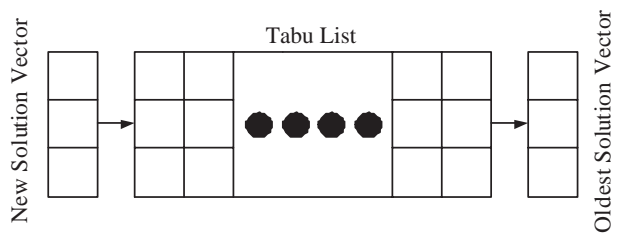

Fig. 4. Mechanism of Tabu List.

In general, if the size of TL is too small, the cycling of solution occurs in the search process. On the other hand, if the size is too large, the search process is in less diversity. Hence, the appropriate size in our applications is between 7 and 30 .

For each generation counter $g$, the tabu list size $(T L S)$ must be satisfied under the condition, $0 \leq T L S \leq \tau(g)$. $\tau(g)$ is the maximum allowable of tabu list size (to be discussed in subsection III-E). The tabu restriction for $k$-th individual, $k=1, \ldots, N P$ can be expressed as:

$$
\begin{aligned}
\sqrt{\sum_{i=1}^{N}\left(\frac{P_{i}^{k, \text { new }}-P_{i}^{\text {tabu }, t}}{P_{i, \max }-P_{i, \min }}\right)^{2}} & <\left(d_{0} \cdot \eta^{g} \triangleq d_{t a b u}\right), \\
t & =1, \ldots, T L S,
\end{aligned}
$$

where, $d_{t a b u}$ is the tabu distance; $d_{0}$ is the initial value of tabu distance $\left(=5 \times 10^{-4}\right) ; \eta$ is the drop factor $(=0.95) ; P_{i}^{t a b u, t}$ is the $i$-th actual parameter of the $t$-th tabued solution in TL.

At the beginning, a higher $d_{t a b u}$ is used to provide diversification in order to reduce the search effort towards the optimal region. Moreover, the intensification will occur when the generation counter $g$ reaches the maximum generation limit, $g_{\max }$.

\section{Fitness Function Evaluation and Rank Selection with Elitism}

The fitness function is evaluated based on a distance term and an objective function term. It can be formulated by:

$$
\begin{aligned}
F_{k}= & 4 \cdot N P-\left(R C_{k}+\alpha \cdot R D_{k}\right), \\
& k=1, \ldots, 2 \times N P,
\end{aligned}
$$

where,

$F_{k}: \quad$ the fitness score of the $k$-th individual, $k=1, \ldots, 2 \times$ $N P$;

$\alpha$ : an adaptive decay scale, which can be obtained from $(15)$; 
$R C_{k}$ : the integer rank score of $C_{k}$ of the $k$-th individual can be assigned by the lowest score $(=1)$ for the lowest $C_{k}$ and the highest score $(=2 \times N P)$ for the highest $C_{k}$;

$R D_{k}$ : the integer rank score of $D_{k}$ of the $k$-th individual can be assigned by the lowest score $(=1)$ for the highest $D_{k}$ and the highest score $(=2 \times N P)$ for the lowest $D_{k}$;

$C_{k}$ : the objective value of the $k$-th individual;

$D_{k}$, the summation of distance between the $k$-th individual and each visited solution vector in TL, can be expressed as:

$$
D_{k}=\sum_{t=1}^{T L S} \sqrt{\sum_{i=1}^{N}\left(\frac{P_{i}^{k}-P_{i}^{t a b u, t}}{P_{i, \max }-P_{i, \min }}\right)^{2}},
$$

where, $P_{i}^{k}$ is the $i$-th actual parameter of the $k$-th individual. With the rank selection operator, the $N P$ highest fitness score individuals will be chosen as parent individuals for next generation. Those individuals are obtained from a combined population $(2 \times N P)$ of the old parent population and the offspring population. This strategy is used to avoid the premature convergence of solution.

Moreover, if a new parent population for the next generation does not contain the current best individual, the elitism will replace the last individual in a new parent population by the current best individual. This technique guarantees the current best individual surviving until the last generation.

\section{E. Adaptive parameter setting strategies}

1) Determination of $N_{m}$ and $N_{c}$ : The parameters setting for $N_{m}$ and $N_{c}$ must satisfy the condition:

$$
N_{m}+N_{c}=N P \text {, }
$$

where, $0 \leq N_{m} \leq N P$ and $0 \leq N_{c} \leq N P$.

Initially, both parameters are set to $50 \%$ of $N P$. For the next generation, if the best offspring individual is better than the best parent individual by mutation (or crossover) process, the parameter $N_{m}$ (or $N_{c}$ ) for the next generation will be increased by the intensification number $(I)$. $I$ is set to $20 \%$ of $N P . N_{c}$ is the number of arithmetic crossover individuals.

Otherwise, if the best offspring individual does not improve solution quality better than the best parent individual, then both parameter settings of $N_{m}$ and $N_{c}$ for the next generation will be recovered to the old generation parameter settings.

2) Determination of $\alpha, \beta$ and $\tau$ : The parameters setting of $\alpha, \beta$, and $\tau$, represented by $S$, can be formulated by (15). $S_{\Delta}$ is the step size related to each parameter. $C_{\min }(g)$ is an objective value of the best offspring individual at the generation counter $g$.

At the beginning, $S$ is set to the maximum value of parameter setting. For the next generation, $S$ is controlled by the generation counter $g$. In this paper, $\beta_{\min }, \beta_{\max }$, and $\beta_{\Delta}$ are set to $0.005,0.5$, and 0.025 , respectively. $\alpha_{\min }, \alpha_{\max }$, and $\alpha_{\Delta}$ are set to $0.005,0.5$, and 0.025 , respectively. $\tau_{\min }, \tau_{\max }$, and $\tau_{\Delta}$ are set to 7,30 , and 1 , respectively.

\section{F. Hybrid TS/EP Procedure}

The Hybrid TS/EP procedure can be described as follows:

Step 1: Read the system data, and specify the parameter settings of Hybrid TS/EP.

Step 2: Initialize the initial individuals, $X_{k}, k=$ $1, \ldots, N P$ and the design specification. Evaluate the objective function $\left(C_{k}\right)$ in (8), and update tabu list (TL). Step 3: Initialize the generation counter $g$ to zero.

Step 4: Execute Hybrid TS/EP operators as follow:

Step 4.1: Perform the perturbation strategies.

Step 4.1.1: Initialize the individual counter $k$ to one.

Step 4.1.2: Perform the mutation based on TL restriction until the $k$-th offspring individual does not satisfy TL restriction in (11).

Step 4.1.3: If $k<N_{m}$, increase the individual counter $k$ by one and go to Step 4.1.2.

Step 4.1.4: Initialize individual counter $k$ to $N_{m}+$ 1.

Step 4.1.5: Perform the arithmetic crossover based on TL restriction until the $k$-th offspring individual does not satisfy TL restriction in (11).

Step 4.1.6: If $k<N P$, increase the individual counter $k$ by one and go to Step 4.1.5.

Step 4.2: Combine the offspring population and parent population into a single population to evaluate the objective value and fitness of each individual.

Step 4.3: Perform the rank selection with elitism mechanism to update the new parent individuals from a combined population with the old parent population and offspring population for the next generation.

Step 4.4: Perform the adaptive parameter setting strategies and update TL.

Step 4.5: If the generation counter $g$ is less than the maximum generation limit $g_{\max }$, increase generation counter $g$ by one and go to Step 4 .

Step 5: Hybrid TS/EP is terminated and the current best individual is a solution for the robust design.

\section{EXPERIMENTAL RESULTS}

The two-area four-machine power system, as delineated in Fig. 1, is used as the study system. Details of system data are given in [5]. PSSs are installed at all machines. The proposed robust design based Hybrid TS/EP is developed via the MATLAB programming language. The parameters of designed PSSs are determined with the proposed objective function (8) by the design procedure. $N P$ and $g_{\max }$ are set to 100 and 200, respectively. The PSSs (16a)-(16d) are obtained for the robustly designed PSSs when the damping 

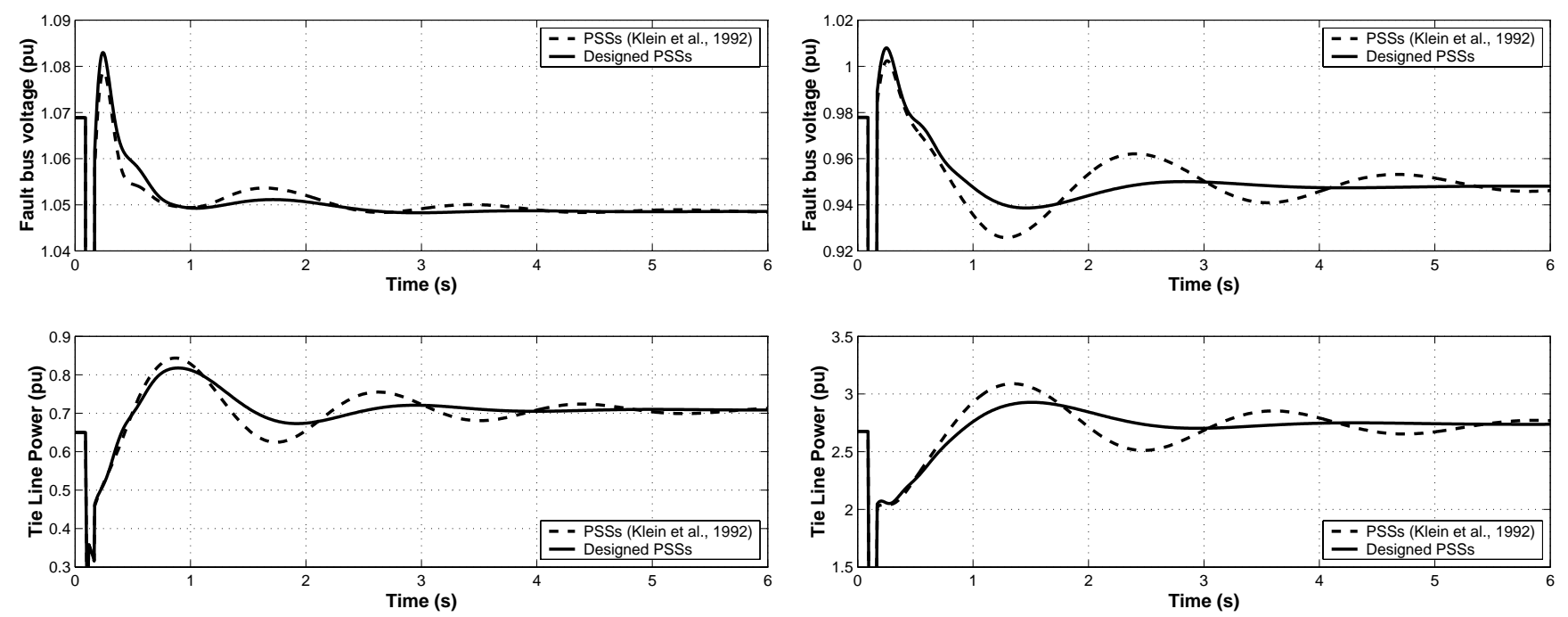

(a) Case 1 .

(b) Case 2 .
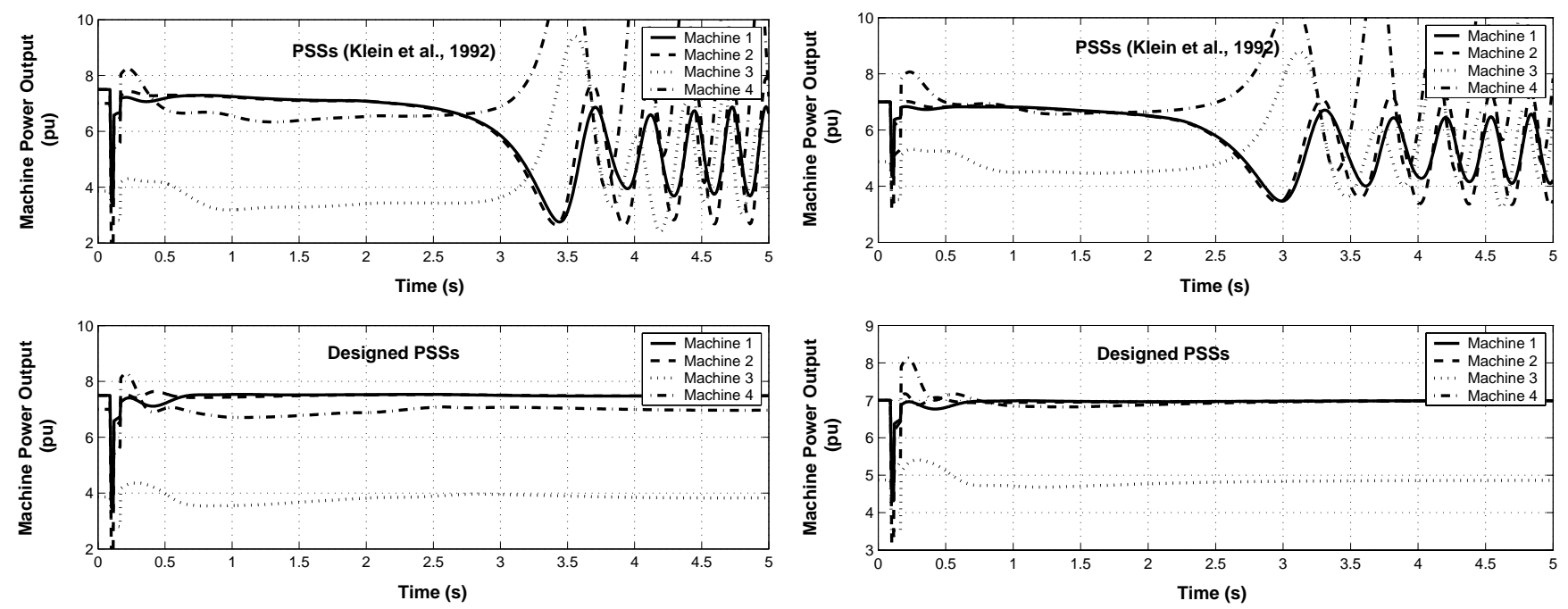

(c) Case 3 .

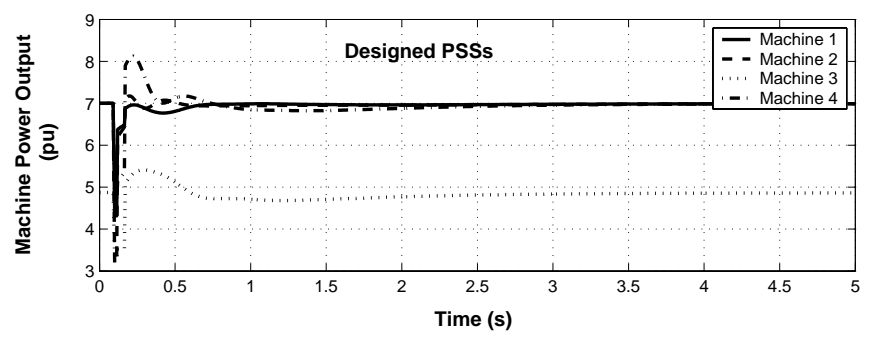

(d) Case 4 .

Fig. 5. System responses results under the four different operating conditions.

factor $\zeta_{\text {desired }}=0.3$ is appointed.

$$
\begin{aligned}
& \text { PSS1 }=11.267 \cdot \frac{10 s}{1+10 s} \cdot \frac{1+0.384 s}{1+0.155 s} \cdot \frac{1+0.112 s}{1+0.091 s}, \\
& \text { PSS }=25.340 \cdot \frac{10 s}{1+10 s} \cdot \frac{1+0.103 s}{1+0.011 s} \cdot \frac{1+0.156 s}{1+0.038 s}, \\
& \text { PSS3 }=16.128 \cdot \frac{10 s}{1+10 s} \cdot \frac{1+0.279 s}{1+0.221 s} \cdot \frac{1+0.389 s}{1+0.387 s}, \\
& \text { PSS4 }=26.381 \cdot \frac{10 s}{1+10 s} \cdot \frac{1+0.112 s}{1+0.010 s} \cdot \frac{1+0.500 s}{1+0.495 s} .
\end{aligned}
$$

The designed PSSs are examined under four different operating conditions as given in Table I. The control effects of designed PSSs are compared with those of PSSs proposed by Klein et al, 1992 in [10]. Note that both designed PSSs and

\begin{tabular}{|c|c|c|}
\hline Case study & $\begin{array}{l}\text { Tie line power, } \\
P_{12}(\mathrm{pu})\end{array}$ & System operating conditions \\
\hline 1. NOC & 1.5 & - \\
\hline 2. HL \& WL & 3 & one circuit $3-101$ is out of servic \\
\hline 3. $\mathrm{HL} \& \mathrm{CP}$ & 5.5 & $20 \%$ constant power load at bus 14 \\
\hline 4. HL, WL \& CP & 4.5 & $\begin{array}{l}\text { one circuit } 3-101 \text { is out of service, } \\
20 \% \text { constant power load at bus } 14\end{array}$ \\
\hline NOC & & $\begin{array}{l}\text { dition; HL = heavy load; } \\
\text { th power load. }\end{array}$ \\
\hline
\end{tabular}
PSSs [10] are designed based on the same operating condition
TABLE I

OpERATING CONDITIONS.

1 in Table I.

Table II shows the eigenvalues and damping ratios of the dominant EMO modes for all case studies. Apparently, the 
damp ratios of EMO modes in case of the designed PSSs are superior to those of PSSs [10]. Table III indicates the values of MSM for each case study. Under various conditions, the MSM, which is the maximum uncertainty bound, of the power system with designed PSSs can maintain at the high value.

TABLE II

DOMINANT MODES AND DAMPING RATIOS.

\begin{tabular}{lccc}
\hline Case & No PSS & PSSs, $[10]$ & Designed PSSs \\
\hline 1. NOC & $-0.08 \pm j 4.05$ & $-0.78 \pm j 4.07$ & $\mathbf{- 1 . 2 7} \pm j 3.55$ \\
& 0.021 & 0.190 & $\mathbf{0 . 3 4}$ \\
2. HL \& WL & $-0.02 \pm j 2.84$ & $-0.46 \pm j 2.83$ & $\mathbf{- 0 . 9 1} \pm j \mathbf{2 . 4 3}$ \\
& 0.007 & 0.159 & $\mathbf{0 . 3 5}$ \\
3. HL \& CP & $-0.05 \pm j 3.39$ & $-0.45 \pm j 3.41$ & $\mathbf{- 0 . 9 3} \pm j 3.05$ \\
& 0.014 & 0.130 & $\mathbf{0 . 2 9}$ \\
4. HL, WL \& CP & $-0.01 \pm j 2.77$ & $-0.39 \pm j 2.77$ & $\mathbf{- 0 . 8 2} \pm j \mathbf{2 . 4 7}$ \\
& 0.003 & 0.138 & $\mathbf{0 . 3 1}$
\end{tabular}

TABLE III

Multiplicative Stability Margins (MSMs).

\begin{tabular}{lcc}
\hline Case & PSSs, [10] & Designed PSSs \\
\hline 1. NOC & 0.9831 & $\mathbf{0 . 8 7 4 4}$ \\
2. HL \& WL & 0.8145 & $\mathbf{0 . 8 5 3 3}$ \\
3. HL \& CP & 0.8489 & $\mathbf{0 . 8 5 7 6}$ \\
4. HL, WL \& CP & 0.7447 & $\mathbf{0 . 8 1 2 6}$ \\
\hline
\end{tabular}

The non-linear simulations [11] are carried out to show the robustness of designed PSSs under four situations as given in Table I. It is assumed that the three-phase fault to ground is applied at bus 101 on one circuit of line 101-13 as shown in Fig. 1. The fault is cleared after $20 \mathrm{~ms}$ and $70 \mathrm{~ms}$ at bus 101 and bus 13, respectively. After the fault is cleared, a faulted circuit of line 101-13 is removed from the network.

As exhibited in Figs. 5(a) and 5(b), the designed PSSs provide more damping effects than PSSs [10]. PSSs [10] completely lose their control effects for operating conditions 3 and 4, as shown in Figs. 5(c) and 5(d). On the other hand, the designed PSSs still retain system stability successfully. This explicitly shows the superior robustness of designed PSSs beyond PSSs [10].

\section{CONCLUSION}

A new robust control design of power system stabilizers in a multimachine power system is proposed in this paper. The Hybrid TS/EP is employed to search for the optimal parameters of PSSs. According to the multiplicative uncertainty model, the MSM can be used to guarantee the robust stability of designed PSSs. Simulation study reveals that the designed PSSs provide the significant damping performance and robust stability against various system uncertainties.

\section{REFERENCES}

[1] Y. Yu, Electric Power System Dynamics. Academic Press, 1983.

[2] Y. Abdel-Magid, M. Abido, and A. Mantawy, "Robust tuning of power system stabilizers in multimachine power systems," IEEE Trans. Power Syst., vol. 15, no. 2, pp. 735-740, 2000.

[3] Y. Abdel-Magid, M. Abido, S. Al-Baiyat, and A. Mantawy, "Simultaneous stabilization of multimachine power systems via genetic algorithms," IEEE Trans. Power Syst., vol. 14, no. 4, pp. 1428-1437, 1999.
[4] M. Abido, "Robust design of multi-machine power system stabilizers using simulated annealing," IEEE Trans. Energy Conversion, vol. 15, no. 3, pp. 297-304, 2000.

[5] G. Rogers, Power System Oscillations (CD-ROM). Kluwer Academic Publishers, 2000.

[6] B. Shahian and M. Hassul, Control System Design using MATLAB. Prentice Hall, 1993.

[7] W.-M. Lin, F.-S. Cheng, and M.-T. Tsay, "An improved tabu search for economic dispatch with multiple minima," IEEE Trans. Power Syst., vol. 17, no. 1, pp. 108-112, Feb. 2002.

[8] M. Gen and R. Cheng, Genetic Algortihms and Engineering Design. New York: John Wiley \& Sons, 1997.

[9] F. Glover and M. Laguna, Tabu Search. London: Kluwer Academic Publishers, 2001.

[10] M. Klein, G. Rogers, S. Moorty, and P. Kundur, "Analytical investigation of factors influencing power system stabilizers performance," IEEE Trans. Energy Conversion, vol. 7, no. 3, pp. 382-388, 1992.

[11] Power System Toolbox: version 2, Cherry Tree Scientific Software, Canada, 2001. 